\title{
Über die morphologische Bedeutung der Arteriae laterales corporis striati bei Reptilien.
}

\author{
Weitere Mitteilungen zur Genese der Basalganglien.
}

Von

Hartwig Kuhlenbeck.

Mit 2 Figuren auf Tafel XIII und 2 Textfiguren.

Die morphogenetische Differenzierung des Zentralnervensystems und seiner einzelnen Abschnitte, wie des Gesamtorganismus überhaupt, steht während der Phylogenese und der Ontogenese unter dem Einfluss verschiedener Faktoren, denen eine formgebende, teilweise kausale Bedeutung zugesprochen werden muss.

Es sind dies zunächst einige allgemeine bioelektrische Erscheinungen, welche überall dort auftreten, wo sich lebendes Protoplasma im Erregungszustand befindet, z. B. im Muskelgewebe, bei Drüsentätigkeit, im wachsenden Embryo. Im Nervengewebe wirken nun diese bioelektrischen Vorgänge richtunggebend, morphogenetisch und histogenetisch, in einer streng gesetzmässigen Weise, die Ariëns-Kappers in seiner Lehre von der Neurobiotaxis formuliert hat.

Ferner ist der Einfluss gewisser Hormone, die vielleicht auch chemotaktisch wirken, nicht ausgeschlossen, obgleich gerade hierüber nur sehr wenig sichere Tatsachen oder Beobachtungen bekannt sind.

Die Entwicklung von Blutgefässen sowie die Blutversorgungsmöglichkeiten, d. h. die Vaskularisationsverhältnisse spielen endlich eine bedeutende morphogenetische Rolle. Man muss sich vorstellen, das diese Faktoren nicht einzeln, sondern gemeinsam einwirken und dass sie in den verschiedenen Abschnitten des Zentralnervensystems in verschiedener Intensität wirksam sind.

Die in der Neurobiotaxis sich kundgebenden bioelektrischen Phänomene sind vor allem im Hirnstamm bei der Morphogenese der motorischen Kerne beteiligt, wie Kappers und seine Schüler gezeigt habep. Bei der Formentwicklung des Endhirns ist, worauf ich selbst hinwies, die Neurobiotaxis als ausschlaggebende Komponente des kortikogenetischen Impulses für die Bildung der Grosshirnrinde bestimmend. 
Meine Studien über die Verhältnisse der Basalganglien bei niederen Wirbeltieren und über die wahrscheinliche Phylogenese dieser Kernmassen liessen mich dagegen viel weniger den Einfluss oder die Wirkung neurobiotaktischer Vorgänge erkennen. Es schien mir, als ob herbei vielmehr die Vaskularisationsverhältnisse als bestimmende Faktoren an erster Stelle kämen. Inwieweit sie aber als kausal oder als kondizional, als primär oder als sekundär zu beurteilen sind, ist m. E. nicht mit Sicherheit zu entscheiden.

Die Basalganglien, so wie wir sie beim Menschen und bei den Saügetieren kennen, sind Gebilde, die für den dritten Grundtyp meiner Einteilung des Wirbeltierendhirns charakteristisch sind. Ihre Entfaltung wird vorgezeichnet durch einen mächtigen Wulst der ventrolateralen Wand des Seitenventrikels. ${ }^{2)}$ Ein grosser Teil dieses Wulstes ist entstanden durch die Einstülpung eines beim zweiten Grundtyp pallial, präkortikal oder kortikal ausgebideten Bezirkes, der Area lateralis Pallii der Amphibien.

Die Entwicklung eines derartigen dicken Wulstes ist natürlich an günstige Blutversorgungsmöglichkeiten durch stärkere Gefässe gebunden, im Gegensatz zur oberflächlich ausgebreiteten Rinde, die bequem durch ein diffuses Netzwerk dünnerer Arterien ernährt werden kann. In der Tat steht auch die Bildung des Wulstes irgendwie in Beziehung (kausal oder kondizional) zum Verlauf der stärksten, das Endhirn versorgenden Arterie, der Arteria cerebralis antero-superior ${ }^{3)}$ bei den Reptilien, welche die einfachsten morphologischen Verhaltnisse des Wirbeltierendhirns vom dritten Grundtyp zeigen. Von der Arteria cerebralis antero-superior dringen nun zahlreiche Äste in das Gehirn ein, um die Zellmassen der

1) Folia Anatomica Japonica, Bd. 2, 1924, S. 350/51.

2) Bei dieser Wulstbildung sind ontogenetisch und phylogenetisch zwei morphologische Elemente beteiligt, die entweder verschmelzen oder von denen das ventrale E.ement vom dorsalen verdrängt wird. Diese Verhältnisse, auf die hier nicht eingegangen werden kann, habe ich an anderer Stelle $\mathrm{zu}$ analysieren versucht ("Weitere Mitteilungen zur Genese der Basalganglien. Über die sogenannten Ganglienhügel.“ Erscheint im Anat. Anz. Bd. 59).

3) Verlauf am Chelonierendhirn abgebildet bei B. Haller, Lehrbuch der vergl. Anatomie, Fig. 829, S. 851 Der Verlauf entspricht dem Sulcus endorhinalis. Auch bei Amphibien habe ich in dieser Linie stärkere, wenn auch mehr diffuse Vaskularisation beobachtet. Auf die Frage endlich, ob das Wachstum des Wulstes durch das Eindringen der Gefässe oder das Wachstum der Gefässe durch die Ausbildung des Wulstes bedingt ist, kann hier nicht eingegangen werden, es sollen nur die morphologischen Zusammenhänge gezeigt werden. 
Basalganglien zu versorgen. Hiervon sind die dorsolateralen Äste, welche in den Abschnitt der grössten Tiefe des Wulstes eintreten, die ansehnlichsten. Elliot Smith, der 1919 auf die Bedeutung dieser (refässe bei den Reptilien (Sphenodon) hinwies, führte den Namen „lateral striate artery" ein.

Für Elliot Smith ist nun diese , lateral striate artery " insofern von grosser morphologischer Bedeutung, als sie bei den Reptilien das Hypopallium vom Palaeostriatum scheiden soll. Das als Neostriatum aufufassende Hypopallium wird nach diesem Autor bei den Saügetieren zum Claustrum, zum Putamen, zum grösseren Teil des Nucleus caudatus und teilweise zu Abschnitten des Mandelkerns. Die Arterie scheidet dann nicht mehr das Palaeostriatum vom Neostriatum, wie bei den Reptilien, sondern löst sich ganz innerhalb des Neostriatum auf, die einzelnen Bestandteile desselben versorgend. ${ }^{4)}$

Diese Auffassung wird von J. B. Johnston (1923) kritisiert. Die morphologische Bedeutung der Arteria lateralis Corporis striati (wie wir sie nennen wollen) werde $u$. a. erheblich beeinträchtigt durch die Tatsache, dass bei der Schildkröte der Nucleus caudatus durch viele Gefässe versorgt werde, die durch die Area pyriformis oder sogar durch den lateralen Rand des ", general pallium " hindurchtreten. ${ }^{5}$ )

In den Mitteilungen von Dart und von Shellshear (1920-21) wird im wesentlichen der Standpunkt Elliot Smiths vertreten.

Das Verhalten der Arteriae laterales corporis striati scheint nun recht gut mit den von mir gedeuteten Homologien der Zellmassen des Basalganglienkomplexes übereinzustimmen und soll daher hier kurz erörtert werden.

Auf den beigegebenen Mikrophotogrammen, darstellend Frontalschnitte durch das Vorderhirn eines Reptils (Testudo graeca) mit besonders ausgeprägtem Wulst des Nucleus epibasalis, sieht man ver-schiedene Gefässe, die von der in der Pia liegenden Arteria cerebralis antero-superior in den Nucleus basalis eindringen (Tafelfig. 1 und 2). Dorsal von diesen kleineren Gefässen zieht eine stärkere Arterie, eine der trteriae laterales corporis striati, in das Innere des Wulstes, (besonders deutlich auf Fig. 2). Sie versorgt aber hier nicht ausschliesslich oder in erster Linie die beiden Abschnitte des Nucleus epibasalis (epl und epm),

4) Journ. comp Neur. Bd. 35,,1923, S. 356.

5) Journ. of Anat. Bd. 53. 1919. S. 290, Abb. 20. 
sondern eher hauptsächlich dem Nucleus centralis, d. h. die das innere des Wulstes ausfüllende, nicht rindenartig angeordnete Zellmasse, welche wahrscheinlich eine Proliferation des dorsolateralen Abschnitts des Nucleus basalis darstellt.

Der Nucleus epibasalis zeigt in den kaudalen zwei Dritteln des Hemisphärenhirns zwei Abschnitte, 1. einen lateralen, der ganz oralwärts mit dem Cortex dorsalis und dem Cortex lateralis, weiter kaudalwärts nur mit dem Cortex lateralis zusammenhängt, 2, einen ventromedialen, welcher den inneren, medialen Anteil der paraventrikulären Strecke des Nucleus epibasalis (Pars paraventricularis) darstellt. Beide Abschnitte sind oft durch eine (auf den Figuren nicht sichtbare) flache Furche geschieden, welche Kappers Sulcus striato-archistriaticus bezeichnet. Bei den Lazertiliern ist der ventromediale Abschnitt erst verhältnismässig weit kaudal als Nucleus sphaericus ausgebildet, bei den Cheloniern aber bereits ziemlich weit frontal, hierdurch kommt bei diesen Tieren die übermässige, sich gewissermassen wieder lateralwärts überschlagende Vorwölbung des Wulstes zustande. Im wesentlichen entspricht der laterale und dorsale Anteil des Nucleus epibasalis der Pars anterior, der ventromediale Anteil der Pars posterior.

Während nun beide Anteile des Nucleus epibasalis Aeste der den Nucleus centralis versorgenden Arteria lateralis corporis striati erhalten, wird die Pars dorsolateralis zum grössten Teil durch kortikale Gefässe, die entweder von der Lamina lateralis oder vom lateralen Abschnitt der Lamina dorsalis her eindringen, mit Blut versehen. Dieses Verhalten entspricht ganz den Angaben, die Johnston für seinen Nucleus caudatus der Schildkröte macht.

Wenden wir uns zu den Homologien. Den dorsolateralen Anteil des Nucleus epibasalis setzen wir, in teilweiser Übereinstimmung mit Elliot Smith, dem Claustrum der Säugetiere gleich. Nur dieser Abschnitt ist es, der sowohl von der Arteria later. corpor. striati wie von Rindengefässen versorgt wird. Den ventromedialen Abschnitt des Epibasalkerns deuten wir als Mandelkernhauptkomplex. Den Nucleus centralis endlich als Striatum sensu strictiori, d. h. als Nucleus caudatus und Putamen. Diese Kernmassen, wie auch der Nucleus basalis (Bett der Stria, Nucleus accumbens) werden ausschliesslich von basalen Gefässen versorgt, deren wichtigstes die Arteria lateralis ist (Textfig. 1). 


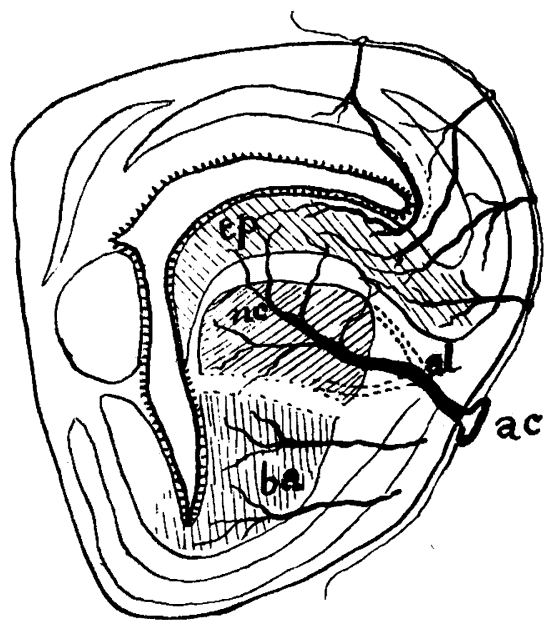

Fig. 1.

Diagramm der Vaskularisation der Basalganglien im Gehirn eines Lazertiliers. ac Arteria cerebralis anterosuperior. ae Arteria lateralis corporis striati. ba Nucleus basalis. ep Nucleus epibasalis. ne Nucleus centralis.

Betrachtet man nun die Vaskularisationsverhältnisse bei den Sä̈getieren, so findet man das Claustrum einerseits von Gefässen versorgt, die bei niederen Saügern durch die Rinde des Lobus pyriformis, bei höheren Saügern infolge der eintretenden Verschiebungen durch die Inselrinde hindurchtreten, also durch Rindengefässe, andererseits durch Äste der im Sulcus endorhinalis und durch das Tuberculum olfactorium eintretenden Gefässe der Basalganglien. Nucleus caudatus und Putamen werden, wie schon bei den Reptilien der Nucleus centralis, ausschliesslich durch diese basalen Gefässe versorgt. Da bei den Saügetieren, besonders beim Menschen, im Schlussplattenmassiv die basalen Ganglien in engste raümliche Beziehungen zu den Kernen des Thalamus und des Hypothalamus treten, werden diese letzteren hier von den Gefässen der Basalganglien aus mit Blut versehen (Arteriae lenticulo-opticae).

Eine wichtige, die innere Kapsel durchsetzende Arterie, die ihr Endgebiet im Wulst des Nucleus caudatus findet, ist Charcot's Arterie der Hirnhämorrhagie (Arteria lenticulo-striata lateralis), sie entspricht einer der Arteriae laterales corporis striati der Reptilien (Textfig. 2).

Andere dieser beim Reptil in einer fronto-kaudalen Reihe angeordneten Arterien finden beim Saügetier hauptsächlich ihr Ausbreitungsgebiet im Claustrum, als Klaustralarterien Shellshears. 


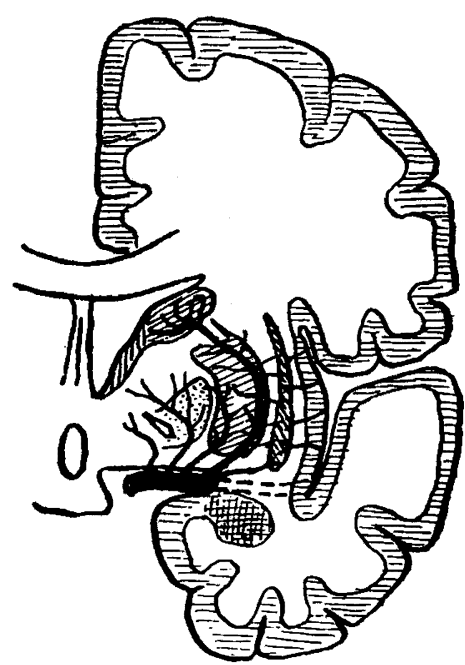

Fig. 2.

Diagramm der Vaskularisation der Basalganglien im menschlichen Gehirn. Die in den Nucleus caudatus eindringende Arterie ist Charcot's Arterie der Hirnhämorrhagie.

\section{Zusammenfassung.}

1. Die für das Saügetiergehirn charakteristische Anordnung der Basalganglien, die sich bereits bei den Reptilien angedeutet findet und die von der Wulstbildung des Nucleus epibasalis ausgeht, ist verbunden mit der Entwicklung besonderer Gefässe, der Arteriae laterales corporis striati.

2. Diese Arterien versorgen hauptsächlich den in Inneren des Epibasaliswulstes gelegenen Nucleus centralis, eine Kernmasse, die bei den Saügetieren das Striatum im engeren Sinne darstellt.

3. Wie bei den Saügetieren wird auch bei den Reptilien das Claustrum teils von basalen (zentralen), teils von kortikalen (oberfächlichen) Arterien versorgt.

4. Einer der Arteriae laterales corporis striati der Reptilien entspricht die Arterie der Hirnhämorrhagie beim Menschen.

Herrn T. Nakano, der in liebenswürdiger Weise die Mikrophotogramme für mich herstellte, möchte ich an dieser Stelle bestens danken.

Tok y o, Juni 1925. 


\section{Literatur.}

1920-21 Dart, A contribution to the morphology of the corpus striatum. Journ. of Anat. 55.

1904 Haller, Lehrbuch der vergleichenden Anatomie. Jena.

1923 Johnston, J. B., Further contributions to the study of the evolution of the forebrain. Journ. Comp. Neur. 35.

1920-21 Kappers, Die vergleichende Anatomie des Nervensystems der Wirbeltiere und des Menschen, Haarlem.

1922 Kuhlenbeck, H., Uber den Ursprung der Grosshirnrinde. Anat. Anz. 55.

1924 Kuhlenbeck, H., Über die Homologien der Zellmassen im Hemisphärenhirn der Wirbeltiere. Fol. Anat. Jap. 2.

1920-21 Shellshear, The basal arteries of the forebrain and their functional significance. Journ. of Anat. 55.

1919 Smith, Elliot, A preliminary note on the morphology of the corpus striatum and the origin of the neopallium. Journ. of Anat. 53.

\section{Figurenerklärung (Tafel XIII).}

Fig. 1. Frontalschnitt durch das Vorderhirn (Zwischen- und Hemisphärenhirn) von Testudo graeca.

Fig. 2. Frontalschnitt durch das Vorderhirn von Testudo graeca bei stärkerer Vergrösserung, die Kernanordungen im Wulst des Nucleus epibasalis und das Endgebiet der Arteria lateralis corporis striati zeigend.

\section{Abkürzungen.}

ac Arteria cerebralis antero-superior. al Arteria lateralis corporis striati. ba Nucleus basalis. ep Nucleus epibasalis. epl Nucleus epibas. later. epm Nucleus epibas. medial. et Eminentia thalami. la Cortex lateralis (Lobus pyriformis). ld Cortex dorsalis (Cortex parahippocampicus). ma Cortex medialis, Pars macrocellularis (Ammonshorn). mi Cortex medialis, Pars microcellularis (Fascia dentata). nc Nucleus centralis. np Primordium neopallii. pa Globus pallidus. 


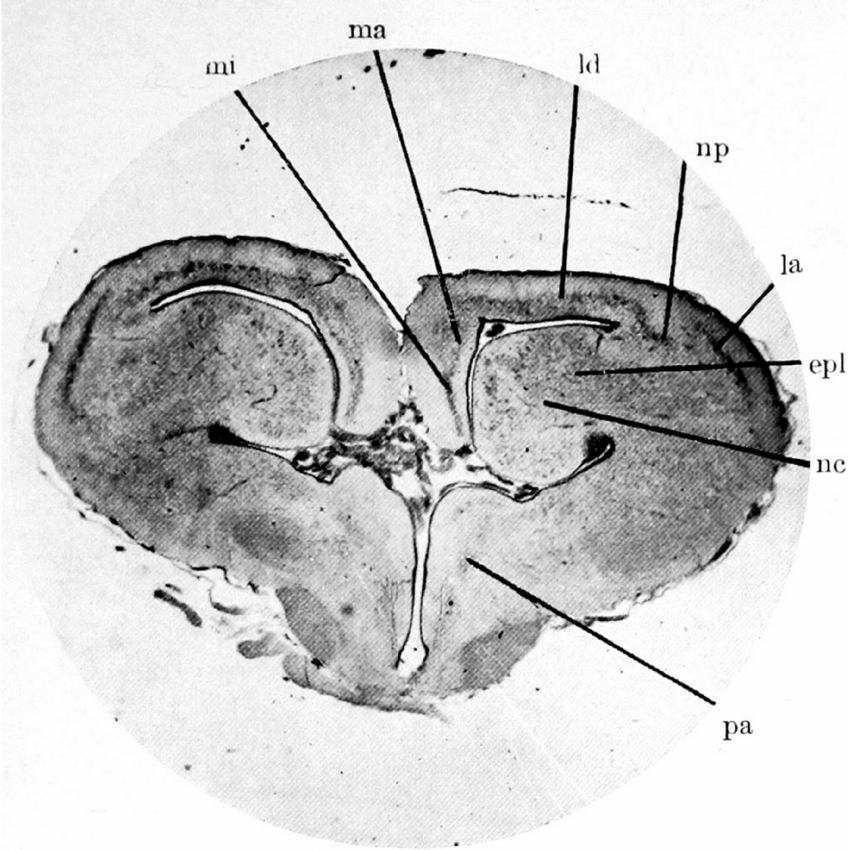

Fig. 1.

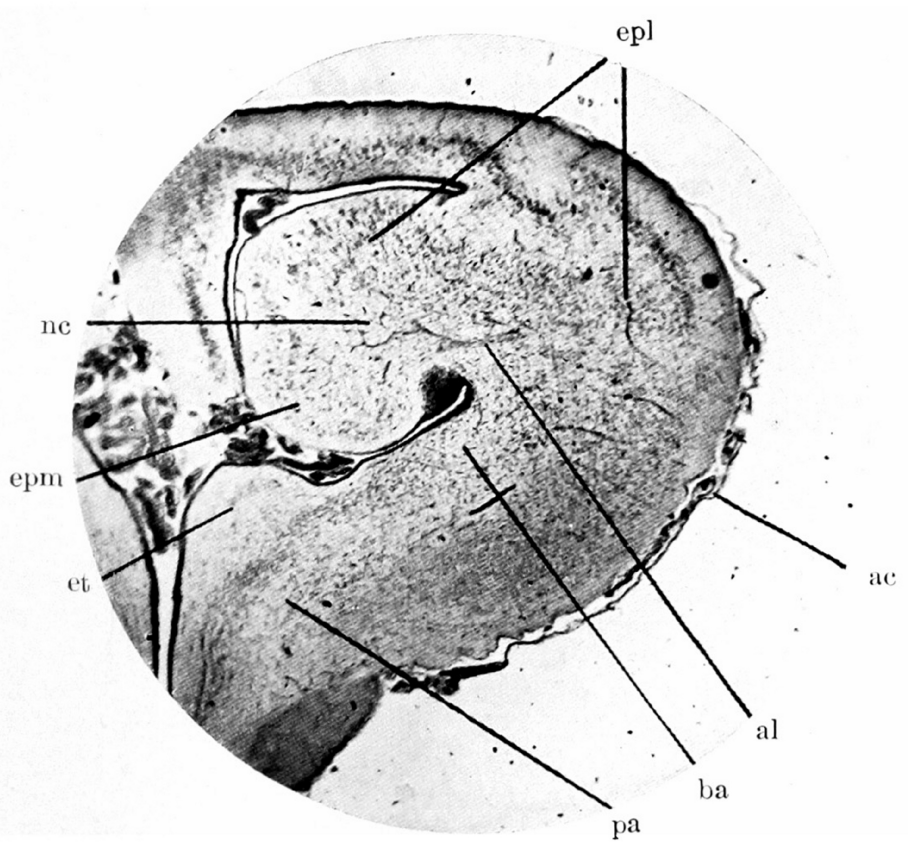

Fig. 2.

H. Kuhlenbeck. 